Rev. Adm. Saúde - Vol. 19, № 75, abr. - jun. 2019

http://dx.doi.org/10.23973/ras.75.164

ARTIGO ORIGINAL

\title{
Sistema de prescrição eletrônica em uma empresa de home care: percepção de médicos e enfermeiros
}

Electronic prescription system in a home care company: perception of physicians and nurses

\section{Lucieno de Moura Santos ${ }^{1}$, James Anthony Falk²}

1. Enfermeiro, mestre em administração. Professor da Faculdade IDE, Recife PE.

2. Doutor em administração pública. Professor adjunto do Departamento de Ciências Administrativas da Universidade Federal de Pernambuco, Recife PE.

\section{RESUMO}

A prescrição médica informatizada, ou prescrição eletrônica, surgiu como meio de mudança na prática médica, onde na adoção dessa tecnologia, vislumbra-se a capacidade do profissional de associá-la no seu dia-a-dia para atingir melhorias para o paciente, para a instituição e para si. O estudo teve por objetivo avaliar a percepção de médicos e enfermeiros sobre a utilização do sistema de prescrição médica eletrônica em uma empresa de home care na cidade do Recife-PE. Trata-se de uma pesquisa de campo com abordagem quantitativa, de natureza exploratória e descritiva. Para a coleta de dados foi elaborado um questionário com respostas apresentadas por uma escala de Likert de cinco possibilidades. Os resultados obtidos foram analisados no software Statistical Package for the Social Sciences (SPSS). Neste, foram feitos os testes de Levine para igualdade de variâncias e o teste $t$ para igualdade das médias. Todos apresentaram significância estatística, pois o valor de $p$ foi menor que 0,005 . Houve evidências estatísticas, ao nível de $5 \%$, de diferenças entre o antes e depois para todas as variáveis. Médicos e enfermeiros perceberam o impacto positivo com a utilização da prescrição médica eletrônica nas variáveis segurança do paciente e da prescrição, bem 
como a redução de erros de prescrição. Onde estas foram também as mais citadas por eles.

Palavras-chave: Tecnologia da informação; Prescrição médica; Assistência domiciliar.

\begin{abstract}
The computerized medical prescription, or electronic prescription, newcomer as a means of attention in the medical hour, where the technology is found, is a capacity of the professional to associate with the attention for the patient, the institution and for themselves. The purpose of this study was to evaluate the medical and nursing care about the use of the medical prescription system in a home care company in the city of Recife-PE. It is a field research with a quantitative approach, exploratory and descriptive in nature. For a data collection, a file with the answers of a Likert scale of five possibilities was elaborated. The results obtained were without software Statistical Package for Social Sciences (SPSS). In this, the Levine tests were made for equality of variables and the test of equality of averages. All values were significant, as the $p$ value was lower than 0.005 . The chance to play at the $5 \%$ level, to differentiate between before and after for all variables. Doctors and doctors have positively warned about the use of the medical prescription with the indication of the medical prescription, as well as the reduction of prescription errors. Where these were also more cited by them.
\end{abstract}

Keywords: Information technology; Medical prescription; Home care.

\title{
INTRODUÇÃO
}

O atendimento domiciliar é uma forma antiga de atenção à saúde, porém as exigências para desempenhar este tipo de atendimento eram poucas e estavam relegadas ao plano doméstico e sem caráter técnico-científico. A função mais atual do home care é a de prestar assistência ao paciente em sua própria residência, levando até ele condições de atendimento e recursos para a melhor evolução possível do seu quadro clínico, dentro dos limites impostos pela doença e dentro de um caráter técnico-científico ${ }^{1}$.

Alguns fatores justificam a adoção do sistema de home care, sejam: o envelhecimento da população, o aumento da necessidade de cuidados médicos em doenças crônicas, aumento do custo de tecnologias diagnósticas e de tratamento, recursos financeiros insuficientes destinados à saúde pelo 
governo, dificuldades das instituições hospitalares, aumento do interesse pelo autocuidado, necessidade de gerenciamento de custo e risco pelos planos de saúde ${ }^{2}$.

Assim, a adoção de tecnologia, em particular dos sistemas de informação, se destaca como elemento integrador e útil para promover a reestruturação das organizações de saúde. Aplicações bem concebidas de tecnologia de informação permitiriam às empresas tornarem-se mais planas, com a eliminação de camadas gerenciais, sendo a própria Tecnologia de Informação um importante elemento na reestruturação não apenas de processos de negócios, mas de toda a empresa ${ }^{3}$.

A tecnologia de informação tem sido um dos maiores impulsionadores do processo de mudança no atendimento holístico ao paciente. Desde a informatização das empresas que prestam serviços de saúde integrando suas áreas internas, passando por redes de clientes e fornecedores, até as tecnologias de ponta - como a utilização de equipamentos sem fio, telemedicina, prontuário eletrônico, entre outros - este setor vem se acelerando cada vez mais, de uma forma nunca observada anteriormente ${ }^{4}$.

O home care vem se adaptando de modo sistematizado à realidade de saúde em termos de modelo assistencial padronizado. Para tanto, um elemento importante no binômio saúde- doença ganha força no desenvolvimento tecnológico: a prescrição médica informatizada ${ }^{5}$.

A integração generalizada e coordenada no uso de software para utilização médica vem ganhando credibilidade e aceitabilidade pela classe, a 'escrita eletrônica' torna-se adequada para registros dos profissionais e para o armazenamento das informações dos diversos pacientes internados ${ }^{6}$.

Um estudo publicado no ano de 2012 na Suíça mostrou que um dos fatores que contribuíam para a ocorrência de erros nas prescrições, bem como a consequente falha na aplicação destas, era a falta de comunicação. Ainda segundo o estudo, as falhas ocorreram por grafias ilegíveis ou incompreensíveis que resultavam em ações prejudiciais para o paciente ${ }^{7}$.

Desta forma surgiu a motivação para estudar o software de prescrição médica eletrônica adaptado especificamente para atender as necessidades de uma empresa de home care na cidade do Recife, avaliando a percepção de médicos e enfermeiros que são os utilizadores tanto na prescrição quanto na checagem respectivamente.

\section{MATERIAL E MÉTODOS}

Trata-se de uma pesquisa de campo com abordagem quantitativa tendo natureza exploratória e descritiva ${ }^{8,9}$. A pesquisa foi realizada em uma empresa de home care na cidade do Recife. Esta possui aproximadamente 300 funcionários e presta assistência a 1.200 pacientes tanto na modalidade de internação domiciliar quanto no programa de gerenciamento de doenças 
crônicas. Não foi objeto do estudo analisar o prontuário eletrônico e sim um componente do prontuário que é a prescrição médica. Para isso é importante ressaltar que antes da utilização da prescrição eletrônica, a empresa adotava tanto o programa Excel para elaboração desta, bem como a prescrição escrita em papel. Este software de prescrição é utilizado pela empresa há 5 anos. A pesquisa propôs-se a entrevistar médicos e enfermeiros que tiveram acesso ao antes e depois da prescrição eletrônica, na tentativa de ter respostas fidedignas quanto a percepção dos utilizadores. Médicos e enfermeiros que não tiveram acesso as práticas anteriores à prescrição eletrônica não foram incluídos no estudo. Desta forma, a amostra foi composta por 9 médicos e 13 enfermeiros.

Para a coleta de dados foi elaborado um questionário com respostas apresentadas por uma escala de Likert de cinco possibilidades. Esta escala é especialmente utilizada em levantamento de atitudes, opiniões e avaliações. Nela pede-se ao respondente que avalie um fenômeno numa escala de, geralmente, cinco alternativas. Avaliam-se objetos ou ações como bons ou ruins. "A avaliação de objetos aplica-se ao respondente, ou concorda-se que objetos ou ações têm uma determinada característica, sendo conveniente formular as perguntas de um conjunto de itens de maneira que seu conjunto possa ser respondido na mesma dimensão" 10 . Os resultados obtidos foram analisados no software Statistical Package for the Social Sciences (SPSS). Neste, foram feitos os testes de Levine para igualdade de variâncias e o teste $t$ para igualdade das médias. Todos apresentaram significância estatística, pois o valor de $p$ foi menor que 0,005 .

\section{RESULTADOS}

Tabela 1. Percepção de médicos e enfermeiros sobre as melhorias apresentadas com a utilização do software de prescrição eletrônica, 2019.

\begin{tabular}{|l|r|}
\hline $\begin{array}{l}\text { Mudanças relatadas por médicos e } \\
\text { enfermeiros }\end{array}$ & $\%$ \\
\hline Redução de erros na prescrição & 21,1 \\
\hline Segurança da prescrição & 18,4 \\
\hline $\begin{array}{l}\text { Segurança para os pacientes } \\
\text { Melhoria na praticidade / agilidade da } \\
\text { prescrição }\end{array}$ & 15,8 \\
\hline $\begin{array}{l}\text { Melhoria no controle de materiais e } \\
\text { medicamentos }\end{array}$ & 13,2 \\
\hline
\end{tabular}




\begin{tabular}{|l|r|}
\hline $\begin{array}{l}\text { Maior organização, detalhamento e clareza } \\
\text { no sistema. }\end{array}$ & 5,3 \\
\hline Melhoria na visualização da prescrição & 5,3 \\
\hline $\begin{array}{l}\text { Melhoria na organização de documentação } \\
\text { para auditoria }\end{array}$ & 2,6 \\
\hline Redução nas dificuldades & 2,6 \\
\hline $\begin{array}{l}\text { Profissionalização dos serviços de } \\
\text { enfermagem }\end{array}$ & 2,6 \\
\hline $\begin{array}{l}\text { Acesso mais rápido à informação e acesso } \\
\text { multidisciplinar }\end{array}$ & 2,6 \\
\hline
\end{tabular}

Foi questionado aos médicos e enfermeiros, quais aspectos foram relevantes com o uso do software de prescrição eletrônica. A Tabela 1 mostra as respostas destes profissionais no que concerne a percepção de melhoria. Estudos já mostraram que um dos objetivos da prescrição eletrônica seria a capacidade do profissional de associá-la ao seu trabalho para atingir melhoria para a empresa ${ }^{11}$.

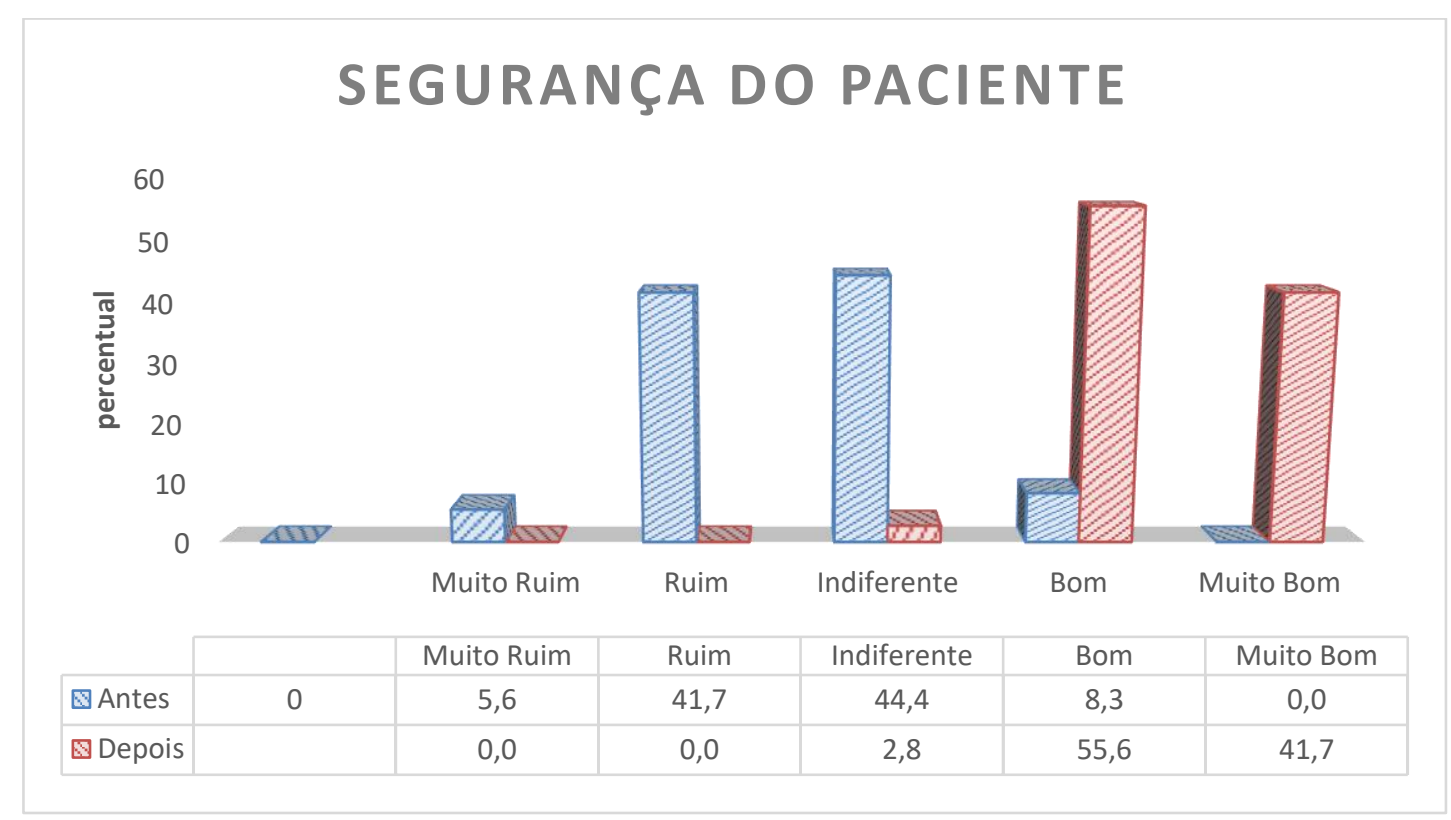


Gráfico 1. Percepção de médicos e enfermeiros acerca da segurança do paciente com a utilização da prescrição médica eletrônica.

De acordo com o Gráfico 1, a variável "segurança do paciente" mostrou-se insatisfatória com o uso em Excel e impressa, onde 5,6\% e 41,7\% dos respondentes informaram ser "muito ruim" e "ruim", respectivamente. Com o novo sistema, $55,6 \%$ e $41,7 \%$ entenderam que o aspecto segurança do paciente passou a ser, respectivamente, "bom" e "muito bom".

O uso da prescrição eletrônica pode melhorar a segurança e reduzir os custos do cuidado ao paciente tendo a adoção dos sistemas de prescrição eletrônica auxiliando na garantia da qualidade e segurança da assistência dos pacientes, bem como de recursos para a prestação de serviços ${ }^{4}$.

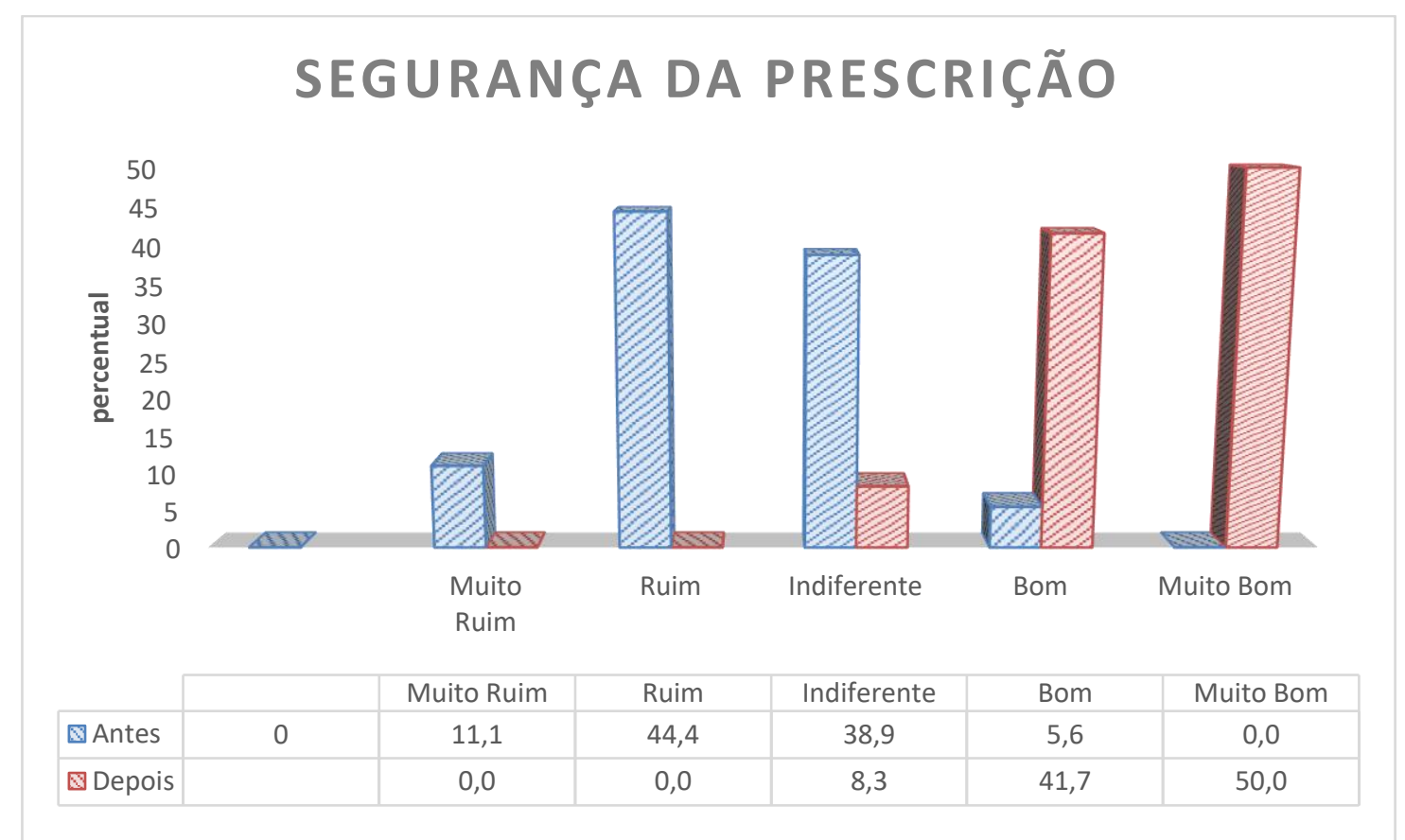

Gráfico 2. Percepção de médicos e enfermeiros acerca da segurança da prescrição com a utilização da prescrição médica eletrônica. 


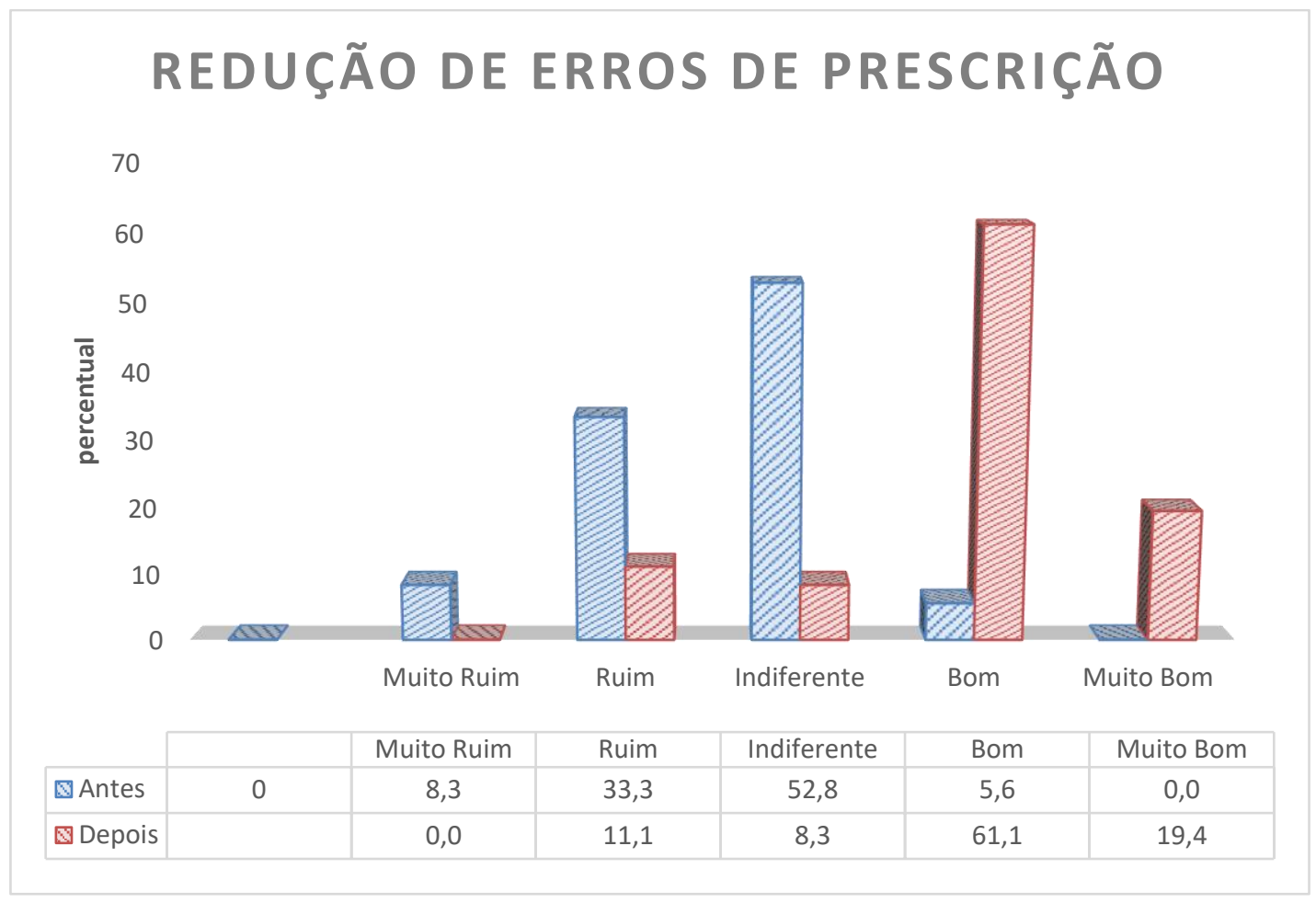

Gráfico 3. Percepção de médicos e enfermeiros acerca da redução de erros de prescrição com a utilização da prescrição médica eletrônica.

A segurança da prescrição está diretamente ligada à redução de erros de prescrição, uma pesquisa publicada em 2005 sinalizou que os erros ocorrem em todas as fases do sistema de medicação: $39 \%$ dos erros ocorrem durante a prescrição, $12 \%$ na transcrição, $11 \%$ na dispensação e $38 \%$ durante a administração. Enfermeiros e farmacêuticos interceptam $86 \%$ dos erros de medicação relacionados a erros de prescrição, transcrição e dispensação, enquanto apenas $2 \%$ são interceptados pelos pacientes, segundo o estudo ${ }^{12}$.

\section{DISCUSSÃO}

Em 2010, foi publicado um estudo onde um dos objetivos foi verificar as vantagens e desvantagens do sistema de prescrição eletrônica utilizado. Segundo as autoras, os funcionários mencionaram a facilidade de leitura dos dados contidos na prescrição médica eletrônica como sua principal vantagem. Os dados do estudo das autoras não reiteram o que foi encontrado nesta pesquisa, pois apenas $5,3 \%$ dos entrevistados disseram ter havido melhoria na visualização da prescrição (Tabela 1) bem como esse mesmo percentual foi apontado na maior organização, detalhamento e clareza no sistema. Cabe ressaltar que a melhoria na visualização é um dos fatores determinantes para a segurança da prescrição, se comparado ao programa anterior ${ }^{13}$. 
A redução dos erros de prescrição foi a variável mais citada, com 21,1\%; em segundo apareceu a melhoria na segurança da prescrição, com 18,4\%, seguida pela melhoria na segurança para os pacientes, com 15,8\% e melhoria na praticidade/agilidade da prescrição, com 13,2\%.

Os dados da Tabela 1 corroboram os achados de outros estudos, pois puderam observar que o software de prescrição eletrônica estudado apresentou melhorias nas variáveis citadas ${ }^{5,13,14,15}$.

Tendo em vista que no caso deste estudo, os médicos e enfermeiros são os operacionalizadores do software no que concerne à inserção das informações e acompanhamento da operacionalização do sistema, foram elaboradas perguntas fechadas para identificar a percepção destes profissionais sobre a utilização do sistema de prescrição eletrônica em detrimento do sistema utilizado anteriormente, que seria uma planilha de Excel ou em algumas situações da prescrição em papel, escrito a próprio punho. As seguintes variáveis foram elencadas: segurança do paciente; segurança da prescrição e redução de erros de prescrição. Estas variáveis foram escolhidas para verificar se as hipóteses são confirmadas ou se destoam em relação aos estudos já publicados $5,11,13,15,16,17$.

De acordo com o Gráfico 2, os entrevistados sinalizaram que a segurança da prescrição era "boa" e "muito boa", representando em percentuais de respectivamente $41,7 \%$ e $50 \%$, totalizando $91,7 \%$. Em relação à redução de erros de prescrição, 61,1\% classificou o aspecto como "bom" e 19,4\% dos entrevistados respondendo que era "muito bom", totalizando $80,5 \%$ de aprovação conforme o Gráfico 3.

Observamos que houve uma percepção de melhoria com a utilização do sistema de prescrição eletrônica nas variáveis estudadas, pudemos constatar que esta percepção de médicos e enfermeiros da empresa de home care foi confirmada com os estudos anteriormente publicados.

\section{CONCLUSÃO}

Médicos e enfermeiros perceberam o impacto positivo com a utilização da prescrição médica eletrônica nas variáveis segurança do paciente e da prescrição, bem como a redução de erros de prescrição. Onde estas foram também as mais citadas por eles.

Estudos sobre avaliação de tecnologias em saúde são recentes. Há alguns anos em muitos países que incorporaram a tecnologia no âmbito dos cuidados com a saúde, estudos são realizados para medir o impacto, obter resultados, tecer estratégias e mensurar custos relacionados às tecnologias empregadas. A tecnologia da informação tem evoluído rapidamente nos últimos anos e levou a mudanças significativas em nossa vida cotidiana pessoal e profissional. Informações e conhecimentos, hoje são muito mais confortáveis e mais disponíveis do que fora há alguns anos atrás. 


\section{REFERÊNCIAS}

1. Braga PP, Sena RR, Seixas CT, Castro EAB, Andrade AM, Silva YC. Oferta e demanda na atenção domiciliar em saúde. Ciência e Saúde Coletiva 21

(3) 903-912. São Paulo, 2016.

2. Leme EO. Sobre o home care. Portal home care.2015 in: http://portalhomecare.com.br/sobre-o-home-care-2/ acesso em 01/05/2019.

3. Nunes AM. O desenvolvimento das tecnologias de informação e comunicação: efeitos na relação médico-paciente em Portugal. Rev Eletron Comun Inf Inov Saúde. Abr.-jun.;12(2): 148-59 2018.

4. Schofield B, Cresswel K, Westbrook J, Slee A, Girling A, Shah S, Coleman J, Sheikh A. The impact of electronic prescribing systems on pharmacists' time and workflow: protocol for a time-andmotion study in English NHS hospitals. BMJ Open 1-5, 2015.

5. Omotosho A, Asanga U, Fakorede A. Electronic Prescription System for Pediatricians. European Scientific Journal 13 (18): 426 -437, 2017.

6. Stichele RV. Propositions pour l'implémentation pratique dans le système informatique medical. Prescription Sous DCl. Bruxelles, 28(275), p. 9, Out. 2007.

7. Nikolic VAM, Hersperger M, Herren D. Erreurs de prescription. Bulletin des médecins suisses. Berne, 44(93), p. 1595-1599, Jun. 2012.

8. Demo P. Metodologia do conhecimento científico. São Paulo: Atlas, p. 120, 2017.

9. Gil AC. Como elaborar projetos de pesquisa. São Paulo, Atlas, $6^{a}$ ed., 184 p. 2017.

10. Carvalho EA. Metodologia de pesquisa em ciências sociais. Athenas, (1) ano. III, jan.-jul, 2014.

11. Cardinal L, Fernandes C. Intervenção farmacêutica no processo da validação da prescrição médica. Rev. Bras. Farm. Hosp. Serv. Saúde, São Paulo 5 (2) 14-19 abr./jun. 2014.

12. Dias JD, Mekaro KS, Tibes CMS, Mascarenhas SHZ. Compreensão de enfermeiros sobre segurança do paciente e erros de medicação. Rev Min Enferm. 18(4): 866-873, 2014.

13. Volpe CRG, Melo EMM, Aguiar LB, Pinho DLM, Stival MM. Fatores de risco para erros de medicação na prescrição eletrônica e manual. Rev. LatinoAm. Enfermagem, (24): 27 - 42, 2016. 
14. Bruyndonckx A, Murovec V. La prescription électronique: une nouvelle obligation légale. Le Spécialiste, Santé, p 115 november 2017.

15. Silva WN, Rached CDA. A implantação da prescrição eletrônica para reduzir dispensações erradas em drogarias do varejo farmacêutico. Anais do VII SINGEP - São Paulo - SP - Brasil - 2018

16. Virk P, Bates DW, Halamka J, Fournier GA, Rothschild JM. Analyzing Transaction Workflows in an e-Prescribing System. AMIA Symposium Proceedings Page, Baltimore, Mar, 26p 2016.

17. Gordon AL, Franklin M, Bradshaw L, Logan P, Elliott R, Gladman JRF. Health status of UK care home residents: a cohort study. Oxford University Press on behalf of the British Geriatrics Society. Published electronically 17 July 2013.

Recebido: 18 de março de 2019. Publicado: 27 de maio de 2019

Correspondência: Lucieno Santos. E-mail: lucienosantos@hotmail.com

Conflito de Interesses: os autores declararam não haver conflito de interesses.

(C) This is an Open Access article distributed under the terms of the Creative Commons Attribution License, which permits unrestricted use, distribution, and reproduction in any medium, provided the original work is properly cited 